\title{
Hydrodynamic profiles for an impurity in an open vibrated granular gas
}

\author{
J. Javier Brey, ${ }^{*}$ M. J. Ruiz-Montero, and F. Moreno \\ Física Teórica, Universidad de Sevilla, Apdo. de Correos 1065, E-41080 Sevilla, Spain
}

(Received 9 November 2005; published 1 March 2006)

\begin{abstract}
The hydrodynamic state of an impurity immersed in a low density granular gas is analyzed. Explicit expressions for the temperature and density fields of the impurity in terms of the hydrodynamic fields of the gas are derived. It is shown that the ratio between the temperatures of the two components, measuring the departure from the energy equipartition, only depends on the mechanical properties of the particles, being therefore constant in the bulk of the system. This ratio plays an important role in determining the density profile of the intruder and its position with respect to the gas, since it determines the sign of the pressure diffusion coefficient. The theoretical predictions are compared with molecular dynamics simulation results for the particular case of the steady state of an open vibrated granular system in the absence of macroscopic fluxes, and a satisfactory agreement is found.
\end{abstract}

DOI: 10.1103/PhysRevE.73.031301

PACS number(s): 45.70.-n, 45.70.Mg, 51.10.+y, 05.20.Dd

\section{INTRODUCTION}

As a consequence of the inelasticity of collisions, granular fluids exhibit a series of behaviors that are in sharp contrast with those of molecular fluids. One of them is the absence of an energy equipartition in a granular mixture of mechanically different species. The granular temperatures of the components of the mixture, defined from the average kinetic energies, are different. Although this possibility was already pointed out many years ago [1], it has not been until recently that a systematic study of the effect has been started. For the homogeneous state of a freely cooling binary mixture of inelastic spheres, an explicit expression for the ratio of temperatures of the two components has been obtained from an approximate solution of the kinetic Enskog equations [2]. The accuracy of this prediction for weak dissipation and low density has been confirmed by molecular dynamics (MD) simulations [3].

The above homogeneous cooling state (HCS) is not accessible experimentally. In order to maintain a granular system fluidized, an external energy supply is required. This is often carried out by means of vibrating walls or external fields, that generate macroscopic gradients in the system because of inelasticity. At a theoretical level, homogeneously driven granular fluids have also been considered. In these models, stochastic forces injecting energy are added, then allowing a system otherwise isolated to reach a steady state. The lack of energy equipartition in the steady state of homogeneously driven granular mixtures has been also analyzed [4], by extending the methods of [2]. Nevertheless, the relationship between this kind of driving and actual experiments is uncertain. The nonequipartition has also been confirmed by MD simulations of simple shear flows [5] and of vibrated granular gases (in the absence of gravity) [6].

Experimental evidence of the coexistence of different temperatures in strongly vibrated granular mixtures has been reported in both two-dimensional [7] and three-dimensional

\footnotetext{
*Electronic address: brey@us.es
}

[8] systems. Moreover, the dependence of the temperature ratio on the several parameters characterizing the system was investigated. Quite surprisingly, it has been observed that the above ratio remains practically constant in the bulk of the system, in spite of the rather large gradients exhibited by each of the partial temperatures.

The lack of energy equipartition has consequences on the expressions of the transport coefficients of a granular mixture [9]. Therefore, it must also affect the shape of the hydrodynamic profiles and, in particular, the density distribution of each of the components in inhomogeneous mixtures. Consequently, it seems clear that the temperature difference can play a role in the segregation phenomena occurring in vibrated granular mixtures $[10,11]$.

Here, the particular case of a low density binary mixture in the tracer limit, i.e., when the mole fraction of one of the components is very low, will be considered. The simplicity of the system allows a detailed and controlled discussion of the hydrodynamic profiles of the tracer component, that can be expressed analytically in terms of those of the other (excess) component. The starting points are the kinetic Boltzmann equations for the mixture, and the analysis is based on the Chapman-Enskog method. The theory is first formulated for a general kind of states and later on particularized for the steady state of an open vibrated system. Then, the theoretical predictions are compared with MD simulation results, and a quite satisfactory agreement is found over a wide range of values of the parameters defining the mechanical properties of the system. A short summary of some of the results presented here was given in Ref. [12].

The plan of the paper is as follows. In Sec. II, some on the fundamental ideas on which the Chapmann-Enskog method, as applied to a one-component system, is based are shortly reviewed. They are used in Sec. III, where an explicit expression for the ratio between the local temperatures of the two components is derived. This ratio only depends on the mechanical properties of the particles, being independent of the gradients of the hydrodynamic fields. An extensive comparison with MD results for a vibrated granular system in the presence of gravity is carried out. The shape of the density profile for the tracer component is investigated in Sec. IV, 
where an expression for the mass flux relative to the local flow is derived to first order in the gradients of the mixture fields (Navier-Stokes order). For the particular case of the steady state of an open vibrated system, an expression for the density profile of the tracer component in terms of the fields of the excess component is obtained. Since analytical forms for these fields are known, the expression can be integrated numerically. Some details of this are given in the Appendix. Again, the theoretical predictions compare well with MD simulation results, although some significant discrepancies show up as the difference in mass of the particles is increased. Special emphasis is put on the relationship between the breakdown of energy equipartition and the relative position of the centers of mass of the two species. Finally, Sec. V contains some further discussion of the results, as well as some comments on their applicability to arbitrary states.

\section{CHAPMAN-ENSKOG DESCRIPTION OF A GRANULAR GAS}

As already mentioned, the aim of this work is to analyze some properties of the hydrodynamic profiles of a dilute granular binary mixture in the tracer limit, i.e., when the mole fraction of one of the species is very small. More precisely, the emphasis is put on the relationship between the profiles of the tracer component and those of the mixture in an arbitrary state. It is assumed that the concentration of the tracer component is so small that its presence does not affect the state of the granular mixture that is, therefore, determined by the state of the other (excess) component. At a kinetic theory level, this implies that the evolution of the oneparticle distribution function of the excess component obeys a closed nonlinear Boltzmann equation. Moreover, in the evolution of the distribution function of the tracer component, the mutual interactions between their particles can be neglected, as compared with the interactions with the particles of the excess component. Consequently, its distribution obeys a linear Boltzmann-Lorentz equation. This is formally equivalent to consider an impurity or intruder immersed in a dilute granular gas, and this will be the terminology used in the following. Let us start by shortly reviewing some basic aspects of the Chapman-Enskog method applied to a onecomponent granular gas that will be needed in the following.

Consider a low density gas composed by smooth inelastic hard spheres $(d=3)$ or disks $(d=2)$ of mass $m$ and diameter $\sigma$. The inelasticity of collisions is modeled by a velocityindependent coefficient of normal restitution $\alpha$, defined in the interval $0<\alpha \leqslant 1$. The particles are submitted to an external force of the gravitational type, so the force acting on each particle has the form $\boldsymbol{F}=-m g_{0} \hat{\boldsymbol{e}}_{z}$, where $g_{0}$ is a constant and $\hat{\boldsymbol{e}}_{z}$ the unit vector in the positive direction of the $z$ axis. It is assumed that in the low density limit, the time evolution of the one-particle distribution function of the gas, $f(\boldsymbol{r}, \boldsymbol{v}, t)$, is accurately described by the nonlinear inelastic Boltzmann equation

$$
\left(\partial_{t}+\boldsymbol{v} \cdot \boldsymbol{\nabla}-g_{0} \frac{\partial}{\partial v_{z}}\right) f(\boldsymbol{r}, \boldsymbol{v}, t)=J[\boldsymbol{v} \mid f, f],
$$

where $J$ is the Boltzmann collision operator describing the scattering of the pairs of particles,

$$
\begin{aligned}
J[\boldsymbol{v} \mid f, f]= & \sigma^{d-1} \int d \boldsymbol{v}_{1} \int d \hat{\boldsymbol{\sigma}} \Theta(\boldsymbol{g} \cdot \hat{\boldsymbol{\sigma}}) \\
& \times \boldsymbol{g} \cdot \hat{\boldsymbol{\sigma}}\left[\alpha^{-2} f\left(\boldsymbol{r}, \boldsymbol{v}^{\prime}, t\right) f\left(\boldsymbol{r}, \boldsymbol{v}_{1}^{\prime}, t\right)-f(\boldsymbol{r}, \boldsymbol{v}, t) f\left(\boldsymbol{r}, \boldsymbol{v}_{1}, t\right)\right] .
\end{aligned}
$$

Here $\Theta$ is the Heaviside step function, $\hat{\boldsymbol{\sigma}}$ is the unit vector pointing from the center of particle 1 to the center of the other colliding particle at contact, and $\boldsymbol{g}=\boldsymbol{v}-\boldsymbol{v}_{1}$ the relative velocity. Moreover, $\boldsymbol{v}^{\prime}$ and $\boldsymbol{v}_{1}^{\prime}$ are the precollisional or restituting velocities, i.e., the initial values of the velocities leading to $\boldsymbol{v}$ and $\boldsymbol{v}_{1}$ following the binary collision defined by $\hat{\boldsymbol{\sigma}}$ :

$$
\begin{gathered}
\boldsymbol{v}^{\prime}=\boldsymbol{v}-\frac{1+\alpha}{2 \alpha}(\boldsymbol{g} \cdot \hat{\boldsymbol{\sigma}}) \hat{\boldsymbol{\sigma}}, \\
\boldsymbol{v}_{1}^{\prime}=\boldsymbol{v}_{1}+\frac{1+\alpha}{2 \alpha}(\boldsymbol{g} \cdot \hat{\boldsymbol{\sigma}}) \hat{\boldsymbol{\sigma}} .
\end{gathered}
$$

Macroscopic fields number density $n(\boldsymbol{r}, t)$, flow velocity $\boldsymbol{u}(\boldsymbol{r}, t)$, and granular temperature, $T(\boldsymbol{r}, t)$, are defined in the usual way as velocity moments of the distribution function,

$$
\begin{gathered}
n(\boldsymbol{r}, t)=\int d \boldsymbol{v} f(\boldsymbol{r}, \boldsymbol{v}, t), \quad n(\boldsymbol{r}, t) \boldsymbol{u}(\boldsymbol{r}, t)=\int d \boldsymbol{v} \boldsymbol{v} f(\boldsymbol{r}, \boldsymbol{v}, t), \\
n(\boldsymbol{r}, t) T(\boldsymbol{r}, t)=\int d \boldsymbol{v} \frac{m V^{2}}{d} f(\boldsymbol{r}, \boldsymbol{v}, t),
\end{gathered}
$$

where $\boldsymbol{V}=\boldsymbol{v}-\boldsymbol{u}$ is the peculiar velocity. Balance equations for the above fields are directly obtained from the Boltzmann equation,

$$
\begin{gathered}
\partial_{t} n+\boldsymbol{\nabla} \cdot(n \boldsymbol{u})=0, \\
\partial_{t} \boldsymbol{u}+\boldsymbol{u} \cdot \boldsymbol{\nabla} \boldsymbol{u}+(m n)^{-1} \boldsymbol{\nabla} \cdot \mathrm{P}+g_{0} \hat{\boldsymbol{e}}_{z}=0, \\
\partial_{t} T+\boldsymbol{u} \cdot \nabla T+2(n d)^{-1}(\nabla \cdot \boldsymbol{q}+\mathrm{P}: \nabla \boldsymbol{u})+T \zeta=0 .
\end{gathered}
$$

The pressure tensor $\mathrm{P}$ and the heat flux $\boldsymbol{q}$ are given by

$$
\begin{aligned}
& \mathrm{P}=\int d \boldsymbol{v} m \boldsymbol{V} \boldsymbol{V} f(\boldsymbol{r}, \boldsymbol{v}, t), \\
& \boldsymbol{q}=\int d \boldsymbol{v} \frac{m}{2} V^{2} \boldsymbol{V} f(\boldsymbol{r}, \boldsymbol{v}, t),
\end{aligned}
$$

respectively. The cooling rate, $\zeta$ in Eq. (7) is due to the energy dissipation in collisions and it is also a functional of the distribution function

$$
\zeta(\boldsymbol{r}, t)=-(n T d)^{-1} \int d \boldsymbol{v} m v^{2} J[\boldsymbol{v} \mid f, f] .
$$

The balance equations (5)-(7) only become a set closed hydrodynamic equations for the fields once $\mathrm{P}, \boldsymbol{q}$, and $\zeta$ are expressed as functionals of them. This requires us to find a solution to the Boltzmann equation such that all the space and time dependence occurs through $n, \boldsymbol{u}$, and $T$. In the Chapman-Enskog procedure, this normal solution is gener- 
ated by expressing it as a series expansion in a formal nonuniformity parameter $\epsilon$,

$$
f=f^{(0)}+\epsilon f^{(1)}+\epsilon^{2} f^{(2)}+\cdots,
$$

where each factor of $\epsilon$ means an implicit gradient of a macroscopic field. Use of the above expansion in the definitions of the fluxes and the cooling rate gives a corresponding expansion for them. Finally, the time derivatives of the fields are also expanded in the gradients, $\partial_{t}=\partial_{t}^{(0)}+\epsilon \partial_{t}^{(1)}+\cdots$, by means of the balance equations. Then, it is

$$
\partial_{t}^{(0)} n=0, \quad \partial_{t}^{(0)} \boldsymbol{u}=0, \quad \partial_{t}^{(0)} T=-T \boldsymbol{\zeta}^{(0)},
$$

where the zeroth order cooling rate $\zeta^{(0)}$ is given by

$$
\zeta^{(0)}(\boldsymbol{r}, t)=-(n T d)^{-1} \int d \boldsymbol{v} m V^{2} J\left[\boldsymbol{v} \mid f^{(0)}, f^{(0)}\right] .
$$

In this way, to zeroth order in $\epsilon$ it is obtained

$$
-\zeta^{(0)} T \frac{\partial}{\partial T} f^{(0)}=J\left[\boldsymbol{v} \mid f^{(0)}, f^{(0)}\right] .
$$

The solution to this equation is chosen such that it verifies the conditions

$$
\begin{gathered}
\int d \boldsymbol{v} f^{(0)}(\boldsymbol{r}, \boldsymbol{v}, t)=n(\boldsymbol{r}, t), \\
\int d \boldsymbol{v} \boldsymbol{v} f^{(0)}(\boldsymbol{r}, \boldsymbol{v}, t)=n(\boldsymbol{r}, t) \boldsymbol{u}(\boldsymbol{r}, t), \\
\int d \boldsymbol{v} \frac{m V^{2}}{d} f^{(0)}(\boldsymbol{r}, \boldsymbol{v}, t)=n(\boldsymbol{r}, t) T(\boldsymbol{r}, t),
\end{gathered}
$$

i.e., it leads to the same hydrodynamic fields as the complete distribution function. This choice is consistent with the solubility conditions for the set of equations generated by the Chapman-Enskog method [13]. Thus $f^{(0)}$ is easily generated from the distribution function for the HCS of a dilute gas [14] by substituting the homogeneous density and temperature fields by the local values $n(\boldsymbol{r}, t)$ and $T(\boldsymbol{r}, t)$, and replacing the velocity $\boldsymbol{v}$ by the peculiar one, $\boldsymbol{V}(\boldsymbol{r}, t)$. Therefore, it has the form [14]

$$
f^{(0)}(\boldsymbol{r}, \boldsymbol{v}, t)=n v_{T}^{-d}(t) \chi\left(V / v_{T}\right),
$$

where $v_{T}^{2}(\boldsymbol{r}, t)=2 T(\boldsymbol{r}, t) / m$ is a local thermal velocity. As a consequence, Eq. (14) can be rewritten as

$$
\frac{\zeta^{(0)}}{2} \frac{\partial}{\partial \boldsymbol{V}} \cdot\left(\boldsymbol{V} f^{(0)}\right)=J\left[\boldsymbol{v} \mid f^{(0)}, f^{(0)}\right] .
$$

Using Eq. (16) it is easily obtained

$$
\begin{gathered}
\partial_{t}^{(1)} n=-\boldsymbol{\nabla} \cdot(n \boldsymbol{u}), \\
\partial_{t}^{(1)} \boldsymbol{u}=-\boldsymbol{u} \cdot \nabla \boldsymbol{u}-(m n)^{-1} \nabla p-g_{0} \hat{\boldsymbol{e}}_{z},
\end{gathered}
$$

$$
\partial_{t}^{(1)} T=-\boldsymbol{u} \cdot \boldsymbol{\nabla} T-\frac{2 T}{d} \boldsymbol{\nabla} \cdot \boldsymbol{u} .
$$

In the last expression, use has been made of the fact that $\zeta^{(1)}=0$ because of symmetry considerations [15]. Moreover, $p=n T$ is the pressure of the gas. The procedure can be continued and Navier-Stokes hydrodynamic equations for a dilute granular gas, with explicit expressions for the transport coefficients in the first Sonine approximation, have been derived $[15,16]$.

\section{TEMPERATURE OF THE IMPURITY}

Let us suppose now that an impurity or intruder of mass $m_{0}$ and diameter $\sigma_{0}$ is added to the system. It is assumed that the presence of the intruder does not affect the state of the gas, so that the one-particle distribution function of the gas particles is still determined by the nonlinear Boltzmann equation (1). Moreover, the macroscopic flow velocity and temperature for the mixture formed by the gas plus the intruder are the same as those for the gas alone, i.e., they are given by Eqs. (4).

The distribution function of the intruder, $f_{0}(\boldsymbol{r}, \boldsymbol{v}, t)$, obeys the linear Boltzmann-Lorentz equation

$$
\left(\partial_{t}+\boldsymbol{v} \cdot \boldsymbol{\nabla}-g_{0} \frac{\partial}{\partial v_{z}}\right) f_{0}(\boldsymbol{r}, \boldsymbol{v}, t)=J_{0}\left[\boldsymbol{v} \mid f_{0}, f\right],
$$

where the collision operator now is

$$
\begin{aligned}
J_{0}\left[\boldsymbol{v} \mid f_{0}, f\right]= & \bar{\sigma}^{d-1} \int d \boldsymbol{v}_{1} \int d \hat{\boldsymbol{\sigma} \Theta}(\boldsymbol{g} \cdot \hat{\boldsymbol{\sigma}}) \\
& \times \boldsymbol{g} \cdot \hat{\boldsymbol{\sigma}}\left[\alpha_{0}^{-2} f_{0}\left(\boldsymbol{r}, \boldsymbol{v}^{\prime \prime}, t\right) f\left(\boldsymbol{r}, \boldsymbol{v}_{1}^{\prime \prime}, t\right)\right. \\
& \left.-f_{0}(\boldsymbol{r}, \boldsymbol{v}, t) f\left(\boldsymbol{r}, \boldsymbol{v}_{1}, t\right)\right] .
\end{aligned}
$$

Here, $\bar{\sigma}=\left(\sigma+\sigma_{0}\right) / 2$ and $\alpha_{0}$ is the coefficient of normal restitution for impurity-gas collisions. The precollisional velocities in this case are given by

$$
\begin{gathered}
\boldsymbol{v}^{\prime \prime}=\boldsymbol{v}-\frac{\left(1+\alpha_{0}\right) \Delta}{\alpha_{0}}(\boldsymbol{g} \cdot \hat{\boldsymbol{\sigma}}) \hat{\boldsymbol{\sigma}}, \\
\boldsymbol{v}_{1}^{\prime \prime}=\boldsymbol{v}_{1}+\frac{\left(1+\alpha_{0}\right)(1-\Delta)}{\alpha_{0}}(\boldsymbol{g} \cdot \hat{\boldsymbol{\sigma}}) \hat{\boldsymbol{\sigma}},
\end{gathered}
$$

with $\Delta=m /\left(m+m_{0}\right)$. The number density for the intruder is

$$
n_{0}(\boldsymbol{r}, t)=\int d \boldsymbol{v} f_{0}(\boldsymbol{r}, \boldsymbol{v}, t) .
$$

The Chapman-Enskog procedure when applied to a mixture, assumes the existence of a normal solution of the Boltzmann equations for the mixture in which all the space and time dependence of the one-particle distribution function of each of the species occurs through a functional dependence on the hydrodynamic fields of the mixture [13]. These fields can be chosen in different, equivalent ways. Here they are taken to be the concentration of the impurity $x_{0}=n_{0} /\left(n_{0}\right.$ $+n) \rightarrow n_{0} / n$, the pressure $p$, the local flow velocity $\boldsymbol{u}$, and the temperature $T$, i.e., 


$$
f_{0}(\boldsymbol{r}, \boldsymbol{v}, t)=f_{0}\left[\boldsymbol{v} \mid x_{0}(\boldsymbol{r}, t), p(\boldsymbol{r}, t), \boldsymbol{u}(\boldsymbol{r}, t), T(\boldsymbol{r}, t)\right] .
$$

Then, an expansion similar to the one given in Eq. (11) is considered for the distribution function of the impurity, $f_{0}$ $=f_{0}^{(0)}+\epsilon f_{0}^{(1)}+\epsilon^{2} f_{0}^{(2)}+\cdots$. To lowest order in $\epsilon$, Eq. (21) becomes

$$
-\zeta^{(0)}\left(p \frac{\partial}{\partial p}+T \frac{\partial}{\partial T}\right) f_{0}^{(0)}=J_{0}\left[\boldsymbol{v} \mid f_{0}^{(0)}, f^{(0)}\right] .
$$

Taking into account that dimensional analysis requires that $f_{0}^{(0)}$ is of the form

$$
f_{0}^{(0)}=x_{0} \frac{p}{T} v_{T}^{-d} \chi_{0}\left(V / v_{T}\right),
$$

Eq. (26) is seen to be equivalent to

$$
\frac{\zeta^{(0)}}{2} \frac{\partial}{\partial \boldsymbol{V}} \cdot\left(\boldsymbol{V} f_{0}^{(0)}\right)=J_{0}\left[\boldsymbol{v} \mid f_{0}^{(0)}, f^{(0)}\right] .
$$

The solution of this equation is chosen such that

$$
\begin{gathered}
\int d \boldsymbol{v} f_{0}^{(0)}(\boldsymbol{r}, \boldsymbol{v}, t)=\int d \boldsymbol{v} f_{0}(\boldsymbol{r}, \boldsymbol{v}, t)=n_{0}(\boldsymbol{r}, t), \\
\int d \boldsymbol{v} \boldsymbol{v} f_{0}^{(0)}(\boldsymbol{r}, \boldsymbol{v}, t)=n_{0}(\boldsymbol{r}, t) \boldsymbol{u}(\boldsymbol{r}, t), \\
\int d \boldsymbol{v} m V^{2} f_{0}^{(0)}(\boldsymbol{r}, \boldsymbol{v}, t)=\int d \boldsymbol{v} m V^{2} f_{0}(\boldsymbol{r}, \boldsymbol{v}, t)=d n_{0}(\boldsymbol{r}, t) T_{0}(\boldsymbol{r}, t) .
\end{gathered}
$$

The last equality in Eq. (31) defines the local temperature, $T_{0}$, of the intruder. The above requirements are consistent with the solubility conditions of the equations generated by the Chapman-Enskog method. It is worth stressing that Eq. (28) holds independently of the specific form of the hydrodynamic fields of the mixture. Thus the multiplication of that equation by $m_{0} V^{2}$ and integration over $\boldsymbol{V}$ yields

$$
\zeta^{(0)}(\boldsymbol{r}, t)=\zeta_{0}^{(0)}(\boldsymbol{r}, t),
$$

where

$$
\zeta_{0}^{(0)}(\boldsymbol{r}, t)=-\left(n_{0} T_{0} d\right)^{-1} \int d \boldsymbol{V} m_{0} V^{2} \boldsymbol{J}_{0}\left[\boldsymbol{v} \mid f_{0}^{(0)}, f^{(0)}\right]
$$

is the lowest order in the gradients of the cooling rate for the temperature of the intruder.

The evaluation of the cooling rates $\zeta^{(0)}$ and $\zeta_{0}^{(0)}$ requires us to solve Eqs. (14) and (28). This can be done in a systematic way by expanding $f^{(0)}$ and $f_{0}^{(0)}$ in terms of an ensemble of orthogonal polynomials [2]. Here we will consider a leading order approximation that is expected to give quite accurate results, at least for not very strong inelasticity. The zeroth order distributions are approximated by Gaussians,

$$
f^{(0)}(\boldsymbol{r}, \boldsymbol{v}, t)=n\left(\frac{2 \pi T}{m}\right)^{-d / 2} e^{-m V^{2} / 2 T}
$$

$$
f_{0}^{(0)}(\boldsymbol{r}, \boldsymbol{v}, t)=n_{0}\left(\frac{2 \pi T_{0}}{m_{0}}\right)^{-d / 2} e^{-m_{0} V^{2} / 2 T_{0}} .
$$

Note that these expressions are consistent with the conditions (14) and (29)-(31). Employing them, it is straightforward to calculate the cooling rates. The technical details needed to evaluate the integrals have already been discussed several times $[2,4,17]$, and they will not be reproduced here. The result is

$$
\begin{gathered}
\zeta^{(0)^{*}} \equiv \frac{\zeta^{(0)}(\boldsymbol{r}, t)}{n(\boldsymbol{r}, t) \sigma^{d-1} v_{T}(t)}=\frac{\sqrt{2} \pi^{(d-1) / 2}}{\Gamma(d / 2) d}\left(1-\alpha^{2}\right), \\
\zeta_{0}^{(0) *} \equiv \frac{\zeta_{0}^{(0)}(\boldsymbol{r}, t)}{n(\boldsymbol{r}, t) \sigma^{d-1} v_{T}(t)}=\nu_{0}^{*}(1+\phi)^{1 / 2}\left(1-h \frac{1+\phi}{\phi}\right),
\end{gathered}
$$

where $h=m\left(1+\alpha_{0}\right) / 2\left(m+m_{0}\right), \nu_{0}^{*}$ is a dimensionless collision rate,

$$
\nu_{0}^{*}=\frac{8 h \pi^{(d-1) / 2}}{\Gamma(d / 2) d}\left(\frac{\bar{\sigma}}{\sigma}\right)^{d-1}
$$

and $\phi$ is the ratio of the mean square velocities for the intruder and fluid particles,

$$
\phi=\frac{m T_{0}(z)}{m_{0} T(z)} .
$$

Substitution of Eqs. (36) and (37) into Eq. (32) gives

$$
(1+\phi)^{1 / 2}\left(1-h \frac{1+\phi}{\phi}\right)=\frac{\beta}{h},
$$

where the parameter $\beta$ is given by

$$
\beta \equiv \frac{1-\alpha^{2}}{4 \sqrt{2}}\left(\frac{\sigma}{\bar{\sigma}}\right)^{d-1}
$$

The solution of this equation provides the expression for $T_{0}(\boldsymbol{r}, t) / T(\boldsymbol{r}, t)$. There is a unique real solution for all allowed values of $h$ and $\beta$. For elastic collisions $\left(\alpha=\alpha_{0}=1\right)$, the solution is $\phi=m / m_{0}$ and the energy equipartition follows. If only the intruder-gas collisions are inelastic $(\alpha=1)$, the result $\phi=h /(1-h)$ derived by Martin and Piasecki for an inelastic impurity in an equilibrium elastic gas is recovered [18].

The simplicity of Eq. (40) may appear as a surprise, especially taking into account its generality. No particular state of either the gas or the intruder has been considered and, nevertheless, the temperature ratio is given as a function of only the mechanical properties of the particles. The existence of an expression for the temperature ratio is a consequence of the assumption that there is a hydrodynamic description of the mixture in terms of only $x_{0}, p, \boldsymbol{u}$, and $T$. Therefore, although the partial temperatures of the intruder and the gas are different, they are not needed to specify the macroscopic state of the mixture. Nevertheless, the theory provides a relationship to determine them. The fact that Eq. (40) does not involve any of the other hydrodynamic fields follows from the tracer and dilute limits we are considering. 
The above relation between the partial temperatures is not modified when applied to the HCS, consistently with previous results $[17,19]$. This does not imply by itself any kind of local HCS approximation, in the same way as the energy equipartition in molecular systems does not imply local equilibrium, and it is valid independently of the gradients present in the system. On the other hand, the above results do not hold if the characterization of the macroscopic state of the mixture also requires to specify the partial temperatures of the gas and the intruder and, therefore, the set of independent hydrodynamic variables (and also the number of equations) must be expanded. This seems to be the kind of approximation followed in [1].

In order to check the accuracy of the theoretical prediction given by Eq. (40), we have performed MD simulations with an event driven algorithm. The particular situation we have considered is a two-dimensional system confined in a rectangular box of width $W$, open on the top, and submitted to an external field of the gravitational type, as defined above Eq. (1). Periodic boundary conditions are enforced in the direction perpendicular to the field. To maintain the system fluidized, energy is continuously supplied through the wall located at $z=0$, which is vibrating. For simplicity, and given that we are interested in the bulk properties of the system, the wall moves in a sawtooth way, with very small amplitude and high frequency, so that it can be considered that all the particles colliding with the wall find it at $z=0$ and with the same velocity $\boldsymbol{v}_{W}=v_{W} \hat{\boldsymbol{e}}_{z}[20,21]$. The particle-wall collisions are elastic.

Under the above conditions, it has been proven that a one-component granular gas reaches a stationary state with only gradients in the $z$ direction [21]. Nevertheless, this state becomes unstable and develops transversal inhomogeneities when the size of the vibrating wall is larger than a critical value [22]. In fact, the same happens for a closed system in the absence of gravity $[23,24]$. In all the simulations to be reported in the following, the value of $W$ has been taken small enough so the system stays homogeneous in the transversal direction.

The above steady state is highly inhomogeneous. An example is given in Fig. 1, where the density and temperature profiles for a monodisperse system of $N=359$ particles with $\alpha=0.95$ are shown. In order to plot the temperature profile on the same scale as the density one, the former has been scaled with an arbitrary value $T_{A}$. Here and in the following, the units defined by $m=1, \sigma=1$, and $g_{0}=1$ will be used. In these units, the data in the figure correspond to $W=50$ and $v_{W}=5$. These values lead to a fluidized state without transversal inhomogeneities, and with a density low enough as to expect the Boltzmann equation to give an accurate description of the system. It is seen that the density profile exhibits a maximum at which $n \simeq 0.075$. The existence of a density maximum is a general feature of open vibrated systems as long as the number of monolayers at rest is large enough [21]. On the other hand, the temperature profile presents a minimum which is related with the existence of a term proportional to the density gradient in the expression of the heat flux $[21,25]$.

Figures $2-4$ show the profile of the temperature ratio $T_{0}(z) / T(z)$ for different values of the mechanical properties

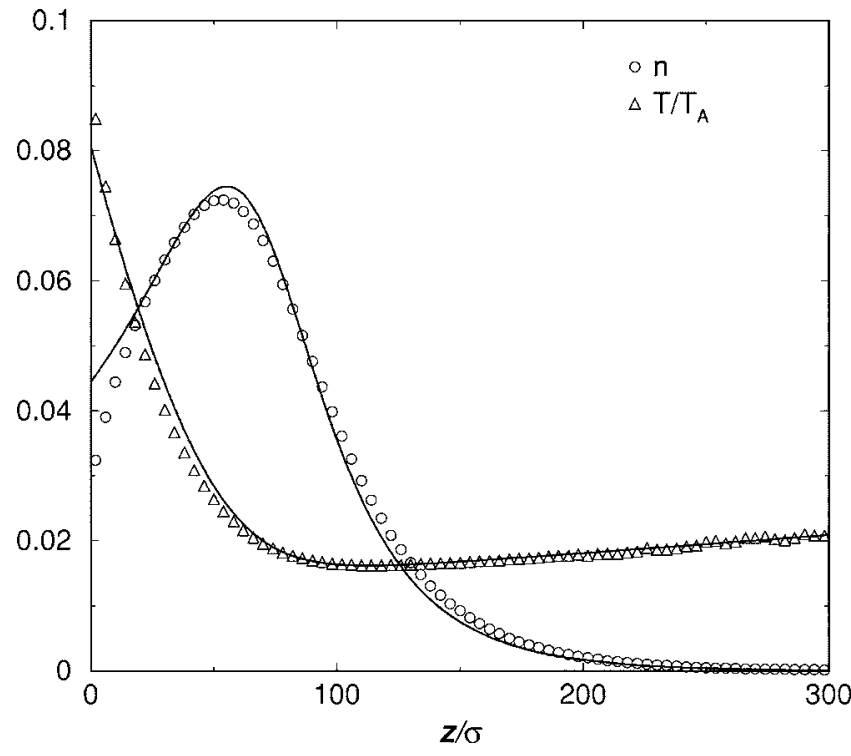

FIG. 1. Density and temperature vertical profiles for a vibrated granular gas in the steady state considered in the text. The number of particles is $N=359$, the restitution coefficient $\alpha=0.95$, and the size of the vibrating wall $W=50 \sigma$. The symbols are from MD simulations and the solid lines the fit to the hydrodynamic prediction discussed in the Appendix.

of the particles. It is observed that, aside statistical fluctuations, the ratio remains practically constant in a wide region of the system, even if $T$ (and $T_{0}$ ) varies significantly. In Fig. 2 , the influence of the inelasticity of the gas-intruder collisions on the temperature ratio is analyzed. As expected, the deviation from the equipartition increases as $\alpha_{0}$ decreases, becoming more different from $\alpha$. The influence of the mass ratio $m_{0} / m$ is studied in Fig. 3, where it is seen that the temperature ratio significantly deviates from unity when $m_{0}$ $=m$, but $\alpha_{0}$ differs significantly from $\alpha$. This is in contrast with previous findings in both experiments [7] and MD

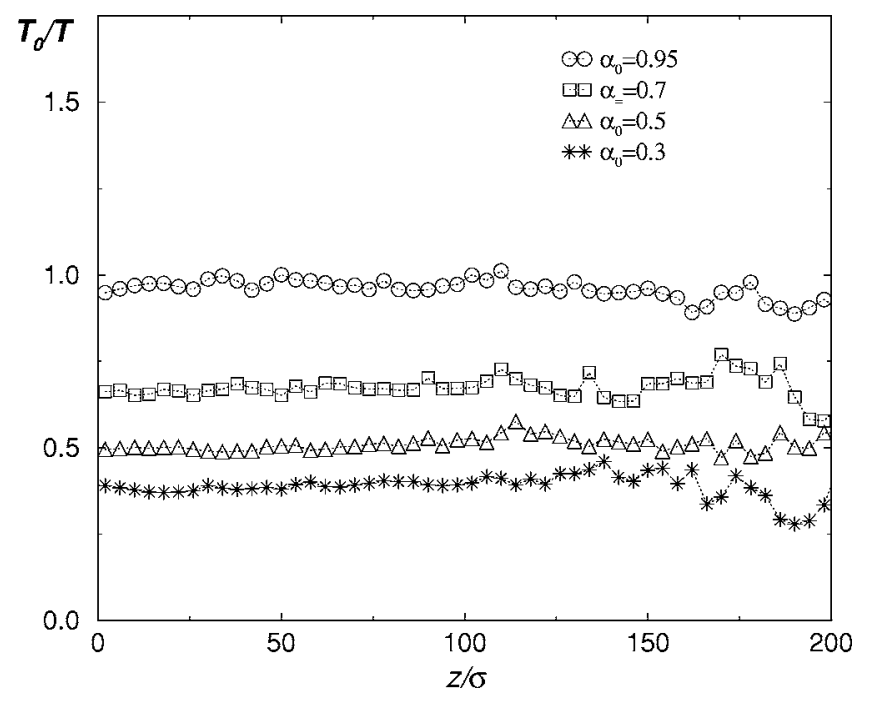

FIG. 2. Temperature ratio profiles $T_{0}(z) / T(z)$ for several values of the restitution coefficient $\alpha_{0}$, as indicated in the figure. The values of the other parameters are $\alpha=0.95, \sigma_{0}=\sigma$, and $m_{0}=m / 2$. 


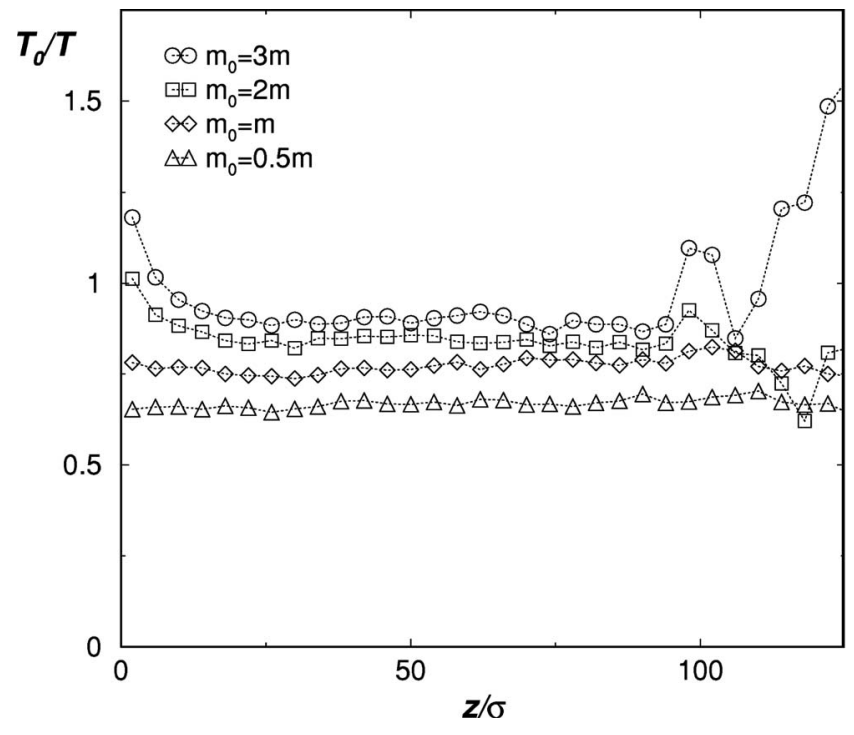

FIG. 3. Temperature ratio profiles $T_{0}(z) / T(z)$ for several values of the mass ratio $m_{0} / m$, as indicated in the figure. The values of the other parameters are $\alpha=0.95, \alpha_{0}=0.7$, and $\sigma_{0}=\sigma$.

simulations [4] of vibrated granular mixtures (not in the tracer limit), probably because the several coefficients of normal restitution in those works were too close to one another as to observe this deviation. The figure also shows that the boundary layer near the vibrating wall, defined as the region in which the hydrodynamic prediction is not verified, increases as the ratio $m_{0} / m$ increases. This can be explained as a consequence of the larger increase of the kinetic energy of the impurity than of the gas particles when colliding with the vibrating wall. In addition, the collisions of the intruder with the gas become less efficient in "thermalizing" the former. The same explanation applies to the strong fluctuations at large heights. Finally, the sensitivity of $T_{0} / T$ on the diameter ratio is illustrated in Fig. 4, from where it can be concluded that it is quite weak.

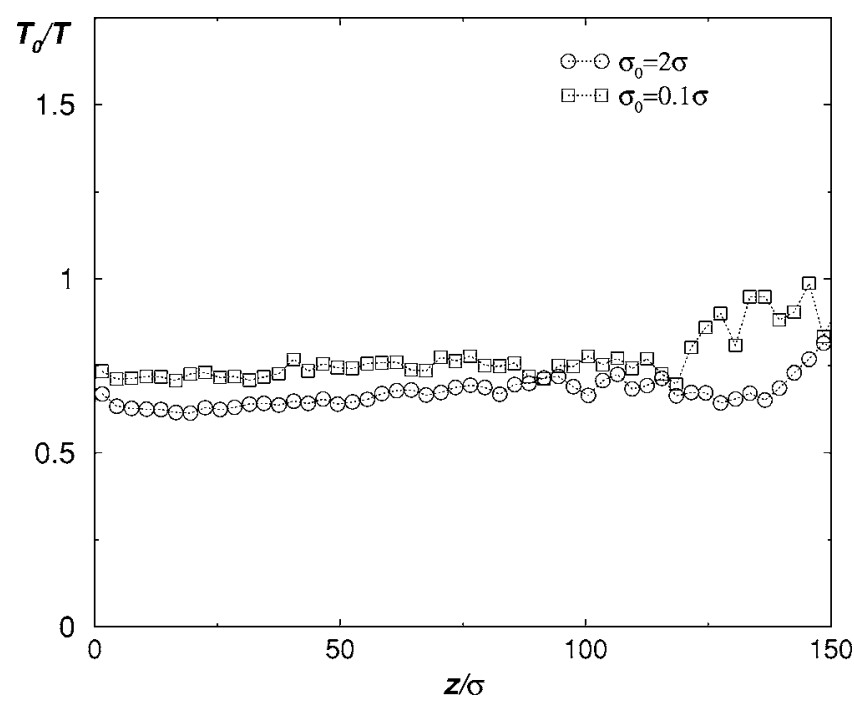

FIG. 4. Temperature ratio profiles $T_{0}(z) / T(z)$ for several values of the diameter ratio $\sigma_{0} / \sigma$, as indicated in the figure. The values of the other parameters are $\alpha=0.95, \alpha_{0}=0.6$, and $m_{0}=m$.

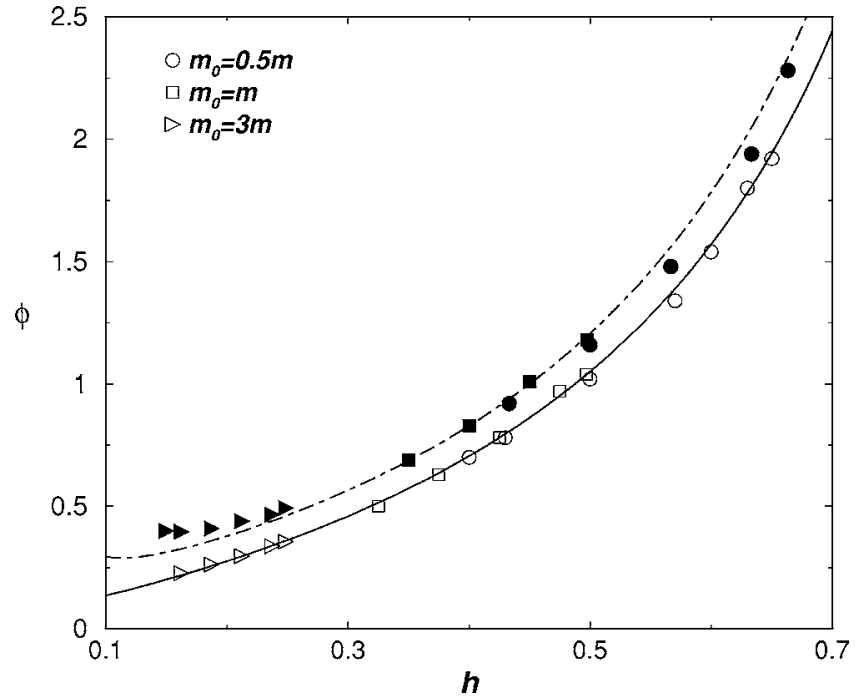

FIG. 5. Ratio of the mean square velocities $\phi$ as a function of the dimensionless parameter $h$ defined in the main text. In all cases $\sigma=\sigma_{0}$. The solid and dashed lines are the theoretical prediction for $\alpha=0.95(\beta \simeq 0.0172)$ and $\alpha=0.8(\beta \simeq 0.0636)$, respectively, while the empty and filled symbols are from the MD simulations for the same two values of $\alpha$. The same symbol is used for simulation results differing only in the value of the restitution coefficient $\alpha_{0}$.

A quantitative comparison between the theoretical prediction given by Eq. (40) and the MD results is presented in Figs. 5 and 6. According with Eq. (40), for a given value of $\beta$, the value of $\phi$ is a function of only the parameter $h$. Then, the comparison will be presented by considering a series of simulations in which the value of the coefficient of restitu-

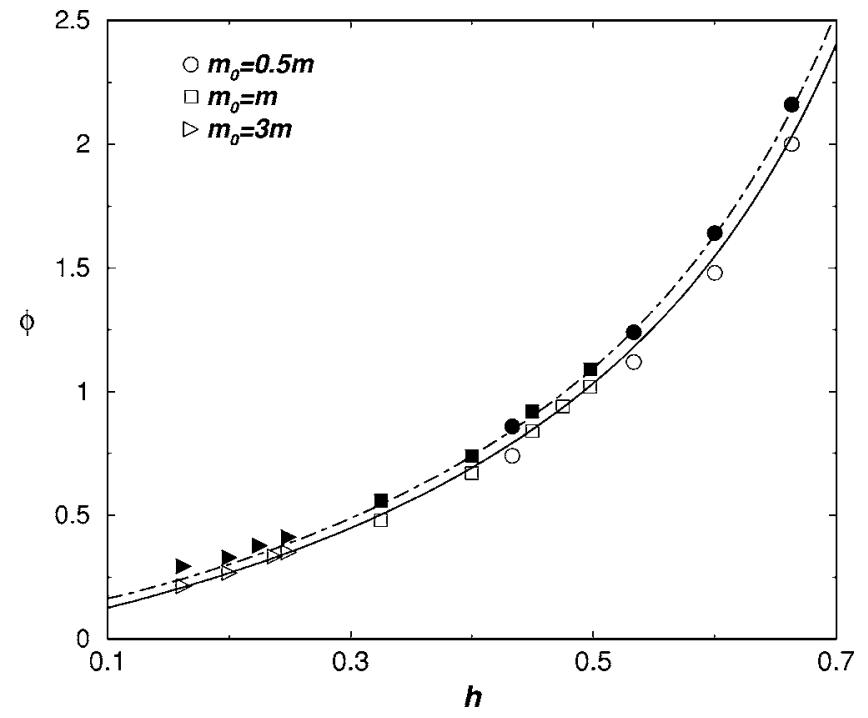

FIG. 6. Ratio of the mean square velocities $\phi$ as a function of the dimensionless parameter $h$ defined in the main text. In all cases $\alpha=0.95$. The solid and dashed lines are the theoretical prediction for $\sigma_{0} / \sigma=2(\beta \simeq 0.0115)$ and $\sigma_{0} / \sigma=0.1(\beta \simeq 0.0313)$, respectively, while the empty and filled symbols are from the MD simulations for those two values of the diameter ratio. The same symbol is used for simulation results differing only in the value of the restitution coefficient $\alpha_{0}$. 
tion of the gas, $\alpha$, and the diameter ratio $\sigma / \sigma_{0}$, and therefore $\beta$ are kept constant, while the mass ratio $m_{0} / m$ and the restitution coefficient $\alpha_{0}$ and, therefore $h$ are changed. In Fig. 5, two series of data, one corresponding to $\sigma_{0}=\sigma, \alpha=0.8(\beta$ $=0.0636)$ and $\sigma_{0}=\sigma, \alpha=0.95(\beta=0.0172)$ are presented. In each case, several values of the mass ratio have been considered, as indicated in the figure. In addition, the value of the coefficient of restitution for gas-intruder collisions has also been varied in the range $0.2 \leqslant \alpha_{0} \leqslant 0.99$. To keep the figure readable, we have used the same symbols for the data corresponding to the same mass ratio although different values of $\alpha_{0}$. A quite good agreement between theory and simulation is observed, especially for the largest value of $\alpha$. The small but systematic discrepancies for $\alpha=0.8$ and $m_{0} / m=3$ are probably due to the failure of the hydrodynamic Navier-Stokes equations to accurately describe the state of the gas, as discussed in [21].

The validity of the theory to describe the dependence on the diameter ratio is checked in Fig. 6. Again, the same symbol is used for the MD results corresponding to situations differing only in the value of the coefficient of restitution $\alpha_{0}$. As before, a weak dependence of the temperature ratio on the diameter of the particles is observed. It can be concluded that Eq. (40) provides an accurate description of the temperature ratio over a wide range of values of the mechanical parameters characterizing both the gas and the intruder.

\section{DENSITY PROFILE FOR THE IMPURITY}

An equation for the number density of the impurity $n_{0}$ is directly obtained from Eq. (21),

$$
\partial_{t} n_{0}+\boldsymbol{\nabla} \cdot\left(n_{0} \boldsymbol{u}\right)+m_{0}^{-1} \boldsymbol{\nabla} \cdot \boldsymbol{j}_{0}=0,
$$

where $\boldsymbol{j}_{0}(\boldsymbol{r}, t)$ is the mass flux for the impurity relative to the local flow $\boldsymbol{u}(\boldsymbol{r}, t)$,

$$
\boldsymbol{j}_{0}(\boldsymbol{r}, t)=m_{0} \int d \boldsymbol{v} \boldsymbol{V} f_{0}(\boldsymbol{r}, \boldsymbol{v}, t) .
$$

The isotropy of the function $\chi_{0}$ implies that the zeroth order of the flux mass for the intruder vanishes, i.e., $\boldsymbol{j}_{0}^{(0)}=0$. Then, we proceed to study the next order, that requires to determine $f_{0}^{(1)}$. To first order in $\epsilon$, Eq. (21) reads

$$
\begin{aligned}
& \partial_{t}^{(0)} f_{0}^{(1)}-J_{0}\left[f_{0}^{(0)}, f^{(1)}\right]-J_{0}\left[f_{0}^{(1)}, f^{(0)}\right] \\
& \quad=-\partial_{t}^{(1)} f_{0}^{(0)}-\boldsymbol{v} \cdot \nabla f_{0}^{(0)}+g_{0} \frac{\partial}{\partial v_{z}} f_{0}^{(0)} .
\end{aligned}
$$

The action of the time derivatives $\partial_{t}^{(0)}$ and $\partial_{t}^{(1)}$ on the hydrodynamic fields is given by Eqs. (12) and (18)-(20), respectively. Then, evaluating the right-hand side of Eq. (44) gives

$$
\begin{aligned}
& \partial_{t}^{(0)} f_{0}^{(1)}-J_{0}\left[f_{0}^{(0)}, f^{(1)}\right]-J_{0}\left[f_{0}^{(1)}, f^{(0)}\right] \\
& \quad=\boldsymbol{A}(\boldsymbol{V}) \cdot \boldsymbol{\nabla} x_{0}+\boldsymbol{B}(\boldsymbol{V}) \cdot \nabla p+\boldsymbol{C}(\boldsymbol{V}) \cdot \nabla T+\mathrm{D}(\boldsymbol{V}): \nabla \boldsymbol{u},
\end{aligned}
$$

where the coefficients of the gradients of the fields on the right-hand side are given by

$$
\begin{gathered}
\boldsymbol{A}(\boldsymbol{V})=-\frac{f_{0}^{(0)}}{x_{0}} \boldsymbol{V}, \\
\boldsymbol{B}(\boldsymbol{V})=-\frac{1}{p}\left(f_{0}^{(0)} \boldsymbol{V}+\frac{T}{m} \frac{\partial f_{0}^{(0)}}{\partial \boldsymbol{V}}\right), \\
\boldsymbol{C}(\boldsymbol{V})=\frac{1}{T}\left[f_{0}^{(0)}+\frac{1}{2} \frac{\partial}{\partial \boldsymbol{V}} \cdot\left(\boldsymbol{V} f_{0}^{(0)}\right)\right] \boldsymbol{V}, \\
\mathrm{D}(\boldsymbol{V})=\boldsymbol{V} \frac{\partial f_{0}^{(0)}}{\partial \boldsymbol{V}}-\frac{1}{d} \boldsymbol{V} \cdot \frac{\partial f_{0}^{(0)}}{\partial \boldsymbol{V}} \mathbf{I} .
\end{gathered}
$$

Here, $\mathbf{I}$ is the unit tensor in $d$ dimensions and $x_{0}$ was defined above Eq. (25). Note that the external field does not occur in Eq. (44). This is because of the particular field we are considering (gravitational type). To proceed, we need the expression of $f^{(1)}$. It has been derived in Ref. [15] and reads

$$
f^{(1)}=\mathcal{B} \cdot \boldsymbol{\nabla} p-\mathcal{C} \cdot \boldsymbol{\nabla} T+\mathcal{D}: \nabla \boldsymbol{u},
$$

where the coefficients $\mathcal{B}, \mathcal{C}$, and $\mathcal{D}$ are functions of the peculiar velocity $\boldsymbol{V}$ and the hydrodynamic fields. Then, the solution of Eq. (45) is of the form

$$
f_{0}^{(1)}=\mathcal{A}_{0} \cdot \boldsymbol{\nabla} x_{0}+\mathcal{B}_{0} \cdot \boldsymbol{\nabla} p+\mathcal{C}_{0} \cdot \boldsymbol{\nabla} T+\mathcal{D}_{0}: \nabla \boldsymbol{u} .
$$

Substitution of Eqs. (50) and (51) into Eq. (45) and identifying coefficients of independent gradients yields

$$
\begin{aligned}
& -\zeta^{(0)}\left[\left(p \frac{\partial}{\partial p}+T \frac{\partial}{\partial p}\right) \mathcal{A}_{0}\right]-J_{0}\left[\mathcal{A}_{0}, f^{(0)}\right]=\boldsymbol{A}, \\
-\zeta_{0} & {\left[\left(p \frac{\partial}{\partial p}+T \frac{\partial}{\partial T}\right)+2\right] \mathcal{B}_{0}-J_{0}\left[f_{0}^{(0)}, \mathcal{B}\right]-J_{0}\left[\mathcal{B}_{0}, f^{(0)}\right] } \\
= & \boldsymbol{B}+\frac{T \zeta^{(0)}}{p} \mathcal{C}_{0}, \\
-\zeta_{0} & {\left[\left(p \frac{\partial}{\partial p}+T \frac{\partial}{\partial T}\right)+\frac{1}{2}\right] \mathcal{C}_{0}-J_{0}\left[f_{0}^{(1)}, \mathcal{C}\right]-J_{0}\left[\mathcal{C}_{0}, f^{(0)}\right] } \\
= & \boldsymbol{C}-\frac{p \zeta^{(0)}}{2 T} \mathcal{B}_{0},
\end{aligned}
$$$$
-\boldsymbol{\zeta}^{(0)}\left[\left(p \frac{\partial}{\partial p}+T \frac{\partial}{\partial T}\right) \mathcal{D}_{0}\right]-J_{0}\left[f_{0}^{(0)}, \mathcal{D}\right]-J_{0}\left[\mathcal{D}_{0}, f^{(0)}\right]=\boldsymbol{D}(\boldsymbol{V}) .
$$

Use of Eq. (51) into Eq. (43) taking into account symmetry considerations, gives the expression for the mass flux of the intruder to first order in the gradients,

$$
\boldsymbol{j}_{0}=-m_{0} D \boldsymbol{\nabla} x_{0}-\frac{m}{T} D_{p} \boldsymbol{\nabla} p-\frac{m n}{T} D^{\prime} \boldsymbol{\nabla} T,
$$

where

$$
D=-\frac{1}{d} \int d \boldsymbol{v} \boldsymbol{V} \cdot \mathcal{A}_{0}
$$

is the diffusion coefficient, 


$$
D_{p}=-\frac{T m_{0}}{m d} \int d \boldsymbol{v} \boldsymbol{V} \cdot \mathcal{B}_{0}
$$

is the pressure diffusion coefficient, and

$$
D^{\prime}=-\frac{T m_{0}}{m n d} \int d \boldsymbol{v} \boldsymbol{V} \cdot \mathcal{C}_{0}
$$

is the thermal diffusion coefficient.

In the following, the quantities $\mathcal{A}_{0}, \mathcal{B}_{0}$, and $\mathcal{C}_{0}$ will be evaluated in the first Sonine approximation, i.e., we consider

$$
\left(\begin{array}{l}
\mathcal{A}_{0} \\
\mathcal{B}_{0} \\
\mathcal{C}_{0}
\end{array}\right) \simeq f_{0}^{(0)} \boldsymbol{V}\left(\begin{array}{l}
a_{0} \\
b_{0} \\
c_{0}
\end{array}\right),
$$

with $f_{0}^{(0)}$ being the Maxwellian given by Eq. (35). Consistently, $\mathcal{B}$ and $\mathcal{C}$ are approximated by

$$
\left(\begin{array}{l}
\mathcal{B} \\
\mathcal{C}
\end{array}\right) \simeq f^{(0)} \boldsymbol{V}\left(\begin{array}{l}
b \\
c
\end{array}\right)
$$

and it happens that both $b$ and $c$ are zero (see Appendix $\mathrm{C}$ in Ref. [15]). Substitution of the above expressions into Eqs. (52)-(54) gives a set of closed equations for $a_{0}, b_{0}$, and $c_{0}$. Multiplication of these equations by $m_{0} \boldsymbol{V}$ and integration over $\boldsymbol{V}$ yields

$$
\begin{gathered}
{\left[-\zeta^{(0)}\left(p \frac{\partial}{\partial p}+T \frac{\partial}{\partial T}\right)+\nu\right] a_{0} n_{0} T_{0}=-\frac{n_{0} T_{0}}{x_{0}},} \\
{\left[-\zeta^{(0)}\left(p \frac{\partial}{\partial p}+T \frac{\partial}{\partial T}\right)-2 \zeta^{(0)}+\nu\right] b_{0} n_{0} T_{0}} \\
=-\frac{n_{0} T_{0}(\phi-1)}{p \phi}+\frac{T \zeta^{(0)} c_{0} n_{0} T_{0}}{p} \\
{\left[-\zeta^{(0)}\left(p \frac{\partial}{\partial p}+T \frac{\partial}{\partial T}\right)-\frac{\zeta^{(0)}}{2}+\nu\right] c_{0} n_{0} T_{0}=-\frac{p \zeta^{(0)} b_{0} n_{0} T_{0}}{2 T},}
\end{gathered}
$$

where

$$
\nu=-\frac{m_{0}}{n_{0} T_{0} d} \int d \boldsymbol{V} \boldsymbol{V} \cdot J_{0}\left[\boldsymbol{V} f_{0}^{(0)}, f^{(0)}\right]
$$

is a collision frequency. It can be easily evaluated by using standard integration techniques with the result

$$
\nu=\frac{\nu_{e}\left(1+\alpha_{0}\right)}{2}(1-\Delta)^{1 / 2}(1+\phi)^{1 / 2},
$$

where

$$
\nu_{e}=\frac{4 \sqrt{2} \pi^{(d-1) / 2}}{\Gamma(d / 2) d} \bar{\sigma}^{d-1} n\left(\frac{T}{m_{0}}\right)^{1 / 2} \Delta^{1 / 2}
$$

is the elastic limit. An expression for $\nu$ for the case $d=3$, valid for arbitrary concentrations of the two components of the mixture and incorporating some non-Gaussian contributions, has been obtained in [9]. Equation (66) is consistent with that result.
From dimensional analysis, it follows that $a_{0} n_{0} T_{0} \propto T^{1 / 2}$, $b_{0} n_{0} T_{0} \propto T^{1 / 2} / p$, and $c_{0} n_{0} T_{0} \propto T^{-1 / 2}$. Then, the temperature and pressure derivatives in Eqs. (62)-(64) can be evaluated and the equations become

$$
\left(\nu-\frac{\zeta^{(0)}}{2}\right) a_{0}=-x_{0}^{-1}
$$

$$
\begin{aligned}
\left(\nu-\frac{3 \zeta^{(0)}}{2}\right) b_{0} & =-\frac{(\phi-1)}{p \phi}+\frac{T \zeta^{(0)}}{p} c_{0}, \\
c_{0} & =-\frac{p \zeta^{(0)}}{2 T \nu} b_{0} .
\end{aligned}
$$

Therefore,

$$
\begin{gathered}
a_{0}=-\left[x_{0}\left(\nu-\frac{\zeta^{(0)}}{2}\right)\right]^{-1}, \\
b_{0}=-\left[\frac{p \phi}{\phi-1}\left(\nu-\frac{3 \zeta^{(0)}}{2}+\frac{\zeta^{(0)^{2}}}{2 \nu}\right)\right]^{-1}, \\
c_{0}=-\frac{p \zeta^{(0)}}{2 T \nu} b_{0} .
\end{gathered}
$$

The expressions of the transport coefficients follow by using the above results into Eqs. (57)-(59)

$$
\begin{gathered}
D=\frac{n T_{0}}{m_{0}}\left(\nu-\frac{\zeta^{(0)}}{2}\right)^{-1} \\
D_{p}=\frac{n_{0} T_{0}(\phi-1)}{m n \phi}\left(\nu-\frac{3 \zeta^{(0)}}{2}+\frac{\zeta^{(0) 2}}{2 \nu}\right)^{-1}, \\
D^{\prime}=-\frac{\zeta^{(0)}}{2 \nu} D_{p} .
\end{gathered}
$$

These expressions are consistent with those reported in Ref. [9] for $d=3$ and without external field.

Let us now particularize the above results for the steady state considered in the previous section, i.e., an open vibrated system with no macroscopic flow and gradients only in the $z$ direction. In this case, Eq. (42) implies that $j_{z}=$ const $=0$, since the intruder mass flux must vanish for $z \rightarrow \infty$. Thus Eqs. (56) and (74)-(76) yield

$$
\begin{aligned}
\left(1-\frac{\zeta^{(0)}}{2 \nu}\right)^{-1} \frac{\partial \ln x_{0}}{\partial z}= & -\frac{\phi-1}{\phi}\left(1-\frac{3 \zeta^{(0)}}{2 \nu}+\frac{\zeta^{(0) 2}}{2 \nu^{2}}\right)^{-1} \\
& \times\left(\frac{\partial \ln p}{\partial z}-\frac{\zeta^{(0)}}{2 \nu} \frac{\partial \ln T}{\partial z}\right) .
\end{aligned}
$$

Using Eqs. (36) and (66), and also the equation determining $\phi$, Eq. (40), it is found

$$
\frac{\zeta^{(0)}}{\nu}=2\left[1-\frac{h(1+\phi)}{\phi}\right] .
$$

This shows that $\zeta^{(0)} / \nu$ is a function of only $\beta$ and $h$, i.e., of the mechanical properties of the particles $[\phi$ is given in 


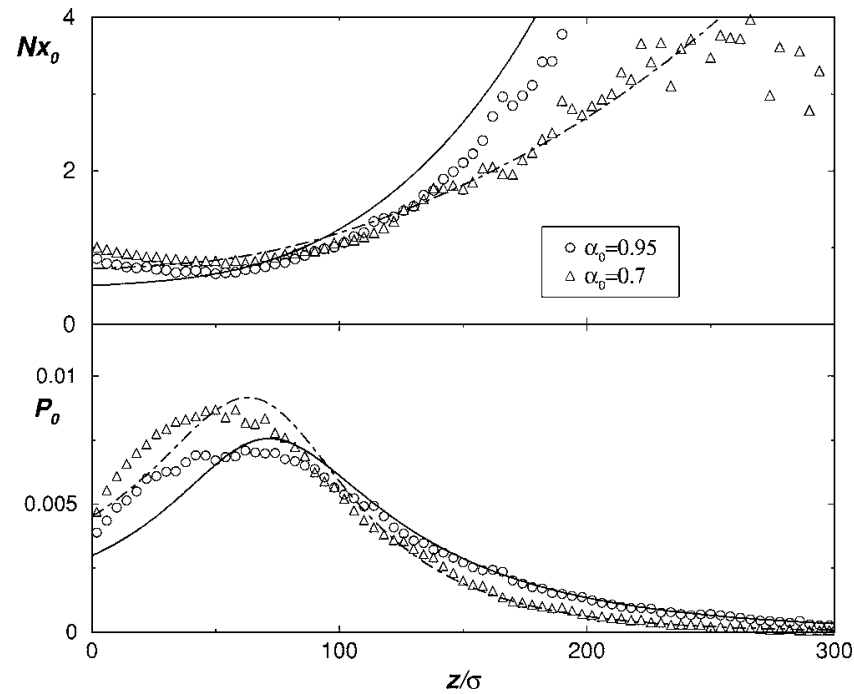

FIG. 7. Normalized molar fraction $N x_{0}$ (upper plot) and density profile $P_{0}(z)$ of the impurity (lower plot) for two different values of $\alpha_{0}$, as indicated in the figure. The symbols are from MD simulations, and the solid (dashed) line the theoretical prediction for $\alpha_{0}$ $=0.95(0.7)$ discussed in the text. The parameters of the gas are $\alpha$ $=0.95, N=359$, and $W=50 \sigma$. The other parameters of the impurity are $m_{0}=m / 2$ and $\sigma_{0}=\sigma$.

terms of them by Eq. (40)], but it does not depend on the hydrodynamic fields. Therefore, Eq. (77) can be expressed in compact form as

$$
\frac{\partial \ln x_{0}}{\partial z}=-\lambda(h, \beta) g(z),
$$

where

$$
\lambda(h, \beta)=\frac{\phi-1}{\phi}\left(1-\frac{\zeta^{(0)}}{2 \nu}\right)\left(1-\frac{3 \zeta^{(0)}}{2 \nu}+\frac{\zeta^{(0) 2}}{2 \nu^{2}}\right)^{-1}
$$

and

$$
g(z)=\frac{\partial \ln p}{\partial z}-\frac{\zeta^{(0)}}{2 \nu} \frac{\partial \ln T}{\partial z} .
$$

The integration of Eq. (79) directly provides an expression for the probability density $P_{0}(z)$ of finding the impurity at height $z$,

$$
P_{0}(z)=C n(z) \exp \left(-\lambda \int_{0}^{z} d z^{\prime} g\left(z^{\prime}\right)\right),
$$

with $C$ being the normalization constant.

The hydrodynamic profiles for the steady state of an open vibrated dilute gas (without impurity) were studied in [21] and the results are shorty summarized in the Appendix. There, it is also indicated how the numerical evaluation of the integral appearing on the right-hand side of Eq. (82) is implemented.

In Figs. 7-9 the theoretical and simulation results for $N x_{0}$ and $P_{0}(z)$ are compared for different values of the parameters. In all cases, those of the gas are the same as in Fig. 1, i.e., $\alpha=0.95, N=359$, and $W=50$, and the diameter ratio is

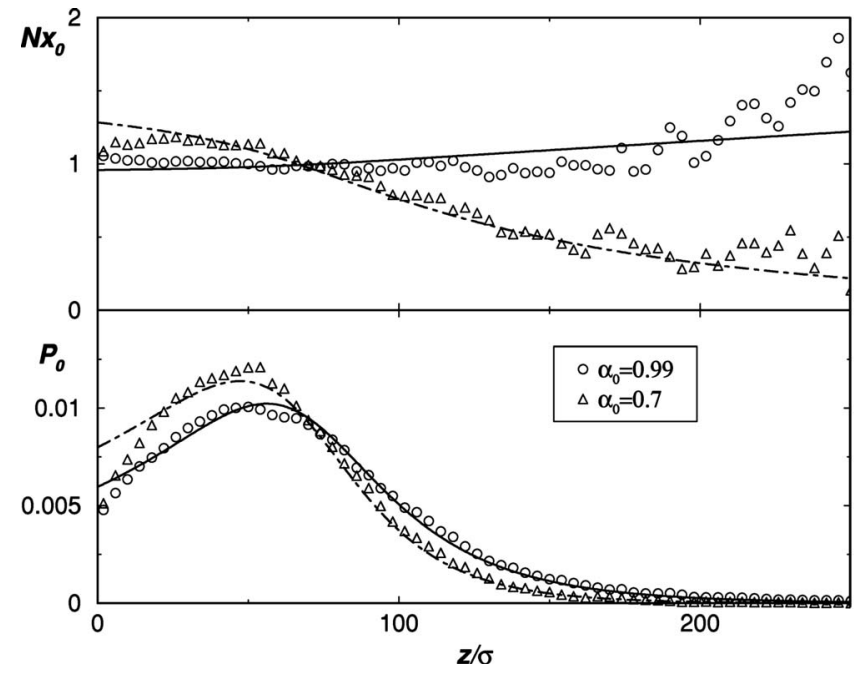

FIG. 8. The same as in Fig. 7, with the only difference that here it is $m_{0}=m$ and the two values of $\alpha_{0}$ considered are 0.99 and 0.7 , as indicated.

$\sigma_{0} / \sigma=1$. On the other hand, in Fig. 7 it is $m_{0} / m=1 / 2$, in Fig. 8, $m_{0} / m=1$, and in Fig. $9, m_{0} / m=3$. Finally, in each of the figures results for two different values of the coefficient of restitution $\alpha_{0}$ are reported.

For the cases reported in Figs. 7 and 8 the agreement between theory and simulation can be considered as quite satisfactory, given that no fitting parameter is being used when constructing the density profile for the impurity from the hydrodynamic profiles of the gas. On the other hand, for $m_{0}=3 m$, Fig. 9 shows strong discrepancies between theory and simulation results. There are several possible causes for it. One is that the heavy intruder is close, on the average, to the vibrating wall and in this region the hydrodynamic description for it is not accurate, as indicated in connection with Fig. 3. In fact, the theoretical prediction for $P_{0}(z)$ is maximum at the wall. Another closely related possible origin of the discrepancy is that we are assuming that the intruder does not affect the hydrodynamic profiles of the gas. This

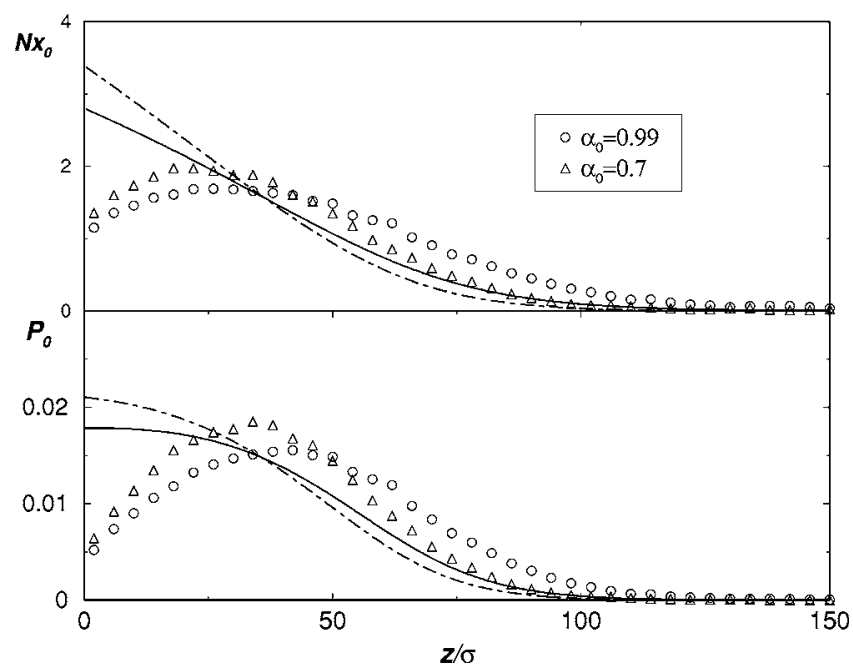

FIG. 9. The same as in Fig. 8, with the only difference that here it is $m_{0}=3 m$. 


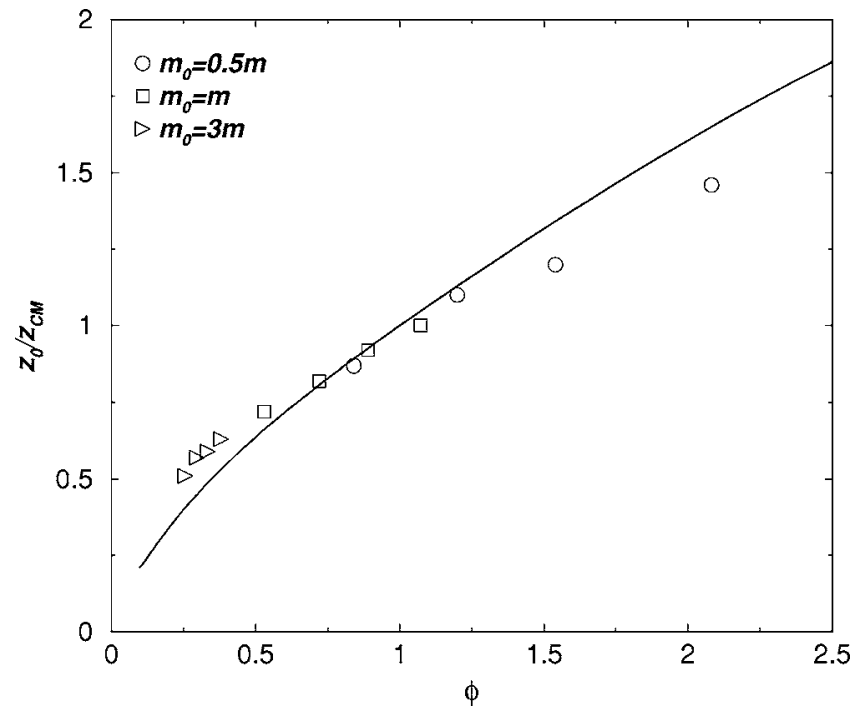

FIG. 10. Ratio between the impurity and the gas center of mass positions vs the ratio of the mean square velocities $\phi$ for systems with $\sigma_{0}=\sigma / 2$. The solid line is the theoretical prediction discussed in the text and the symbols MD simulation results. The same symbol is used for simulations differing only on the value of the restitution coefficient $\alpha_{0}$, which has been varied between 0.3 and 0.95 . The values of the other parameters are given in the text.

may be a bad approximation when the intruder is too massive as compared with the gas particles, especially taking into account that the number of the latter is not very large. We are reminded that this number cannot be increased too much, in order to keep the system fluidized with low density and avoiding the transversal instability mentioned in Sec. III.

We have investigated also the density profile of the intruder for other values of the diameter ratio $\sigma_{0} / \sigma$, namely $\frac{1}{2}$ and 2, and obtained similar results.

From $P_{0}(z)$, the average height of the intruder $z_{0}$ can be computed through

$$
z_{0}=\int_{0}^{\infty} d z z P_{0}(z)
$$

The ratio $z_{0} / z_{c m}$, where $z_{c m}$ is the height of the center of mass of the gas, provides a measurement of the relative position of the intruder with respect to the gas. This ratio is plotted in Fig. 10 as a function of $\phi$ for several systems with $\alpha=0.95$ and $\sigma_{0} / \sigma=\frac{1}{2}(\beta \simeq 0.0230)$. The solid line is the theoretical prediction and the symbols simulation results for different values of the mass ratio and $\alpha_{0}$. Figures 11 and 12 show the same function but for $\sigma_{0} / \sigma=1 \quad(\beta \simeq 0.0172)$ and $\sigma_{0} / \sigma=2$ $(\beta \simeq 0.0115)$.

The agreement between theory and simulation is good in all cases, especially in the region close to $\phi=1$. In fact, for $m_{0} / m=3$ it is better than it could be expected from Fig. 9. This good agreement is not surprising, since we are looking now to the simplest global property of the distribution $P_{0}(z)$. On the other hand, the simulation results for $\sigma_{0}=2 \sigma$ are systematically above the theoretical prediction. In general, it seems that the slope of the simulation results is smaller than

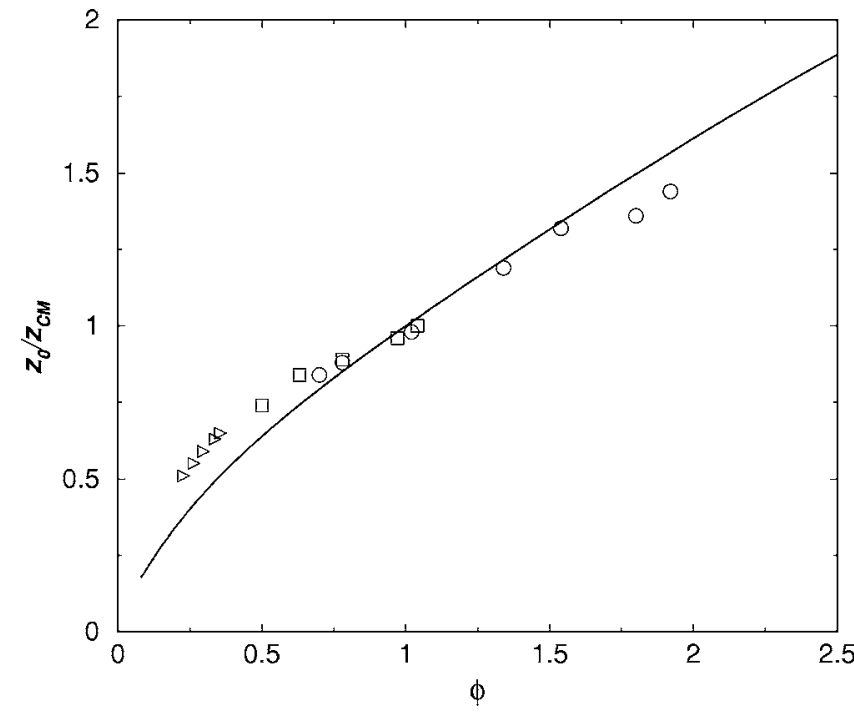

FIG. 11. The same as in Fig. 10, with the only difference that here it is $\sigma_{0}=\sigma$.

the theoretical prediction, although we have no explanation for it. A relevant conclusion to be reached from the above results is the dominant role played by the value of $\phi$ in determining the relative position of the intruder with respect to the gas. In an elastic system, $\phi$ is equal to the mass ratio $m / m_{0}$, but in a granular gas $\phi$ can be larger or smaller than the mass ratio due to the lack of energy equipartition. Consequently, the relative position of an intruder in a granular gas depends in a rather complicated way on the coefficients of restitution of the system, the mass ratio, and the size ratio.

\section{CONCLUSIONS}

In this work, we have studied the hydrodynamic profiles of an impurity immersed in a low density granular gas that is in an arbitrary state. Analytical predictions for the tempera-

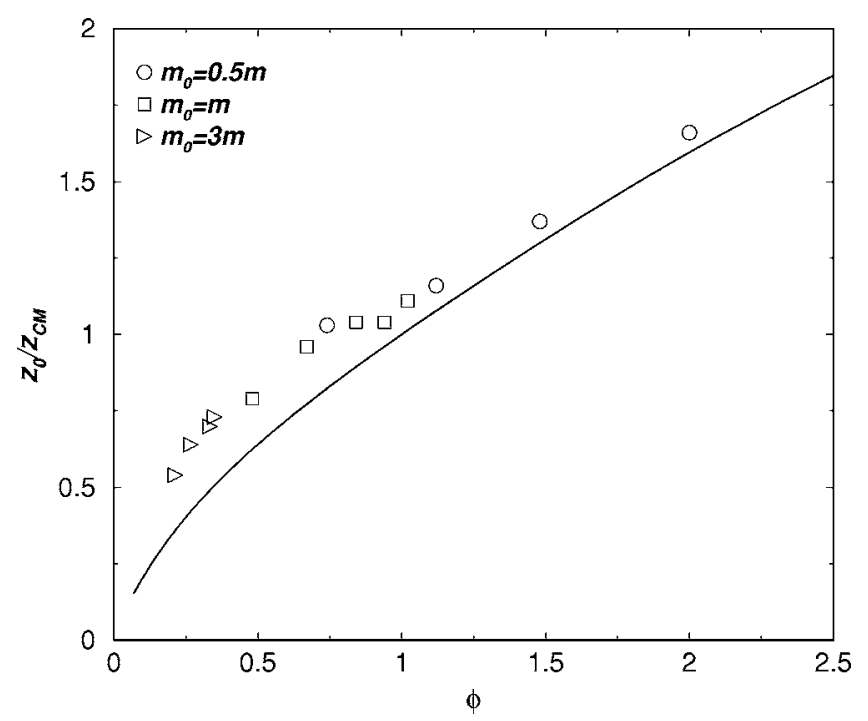

FIG. 12. The same as in Fig. 11, with the only difference that here it is $\sigma_{0}=2 \sigma$. 
ture and density profiles have been derived and compared with molecular dynamics simulations for the particular case of a vibrated system. A satisfactory qualitative and quantitative agreement has been found, although the discrepancies increase as the difference between the masses of the gas particles and the intruder increases. When this is the case, the boundary layer next to the vibrating wall becomes rather wide and, moreover, the intruder influences the density profile of the gas, contrary to the tracer limit assumed in the theory.

The theoretical study is based on the validity of the usual Chapman-Enskog procedure to generate the hydrodynamic equations of the system when starting from the kinetic (Boltzmann) equations. Then, although the results are quite general and can be applied to a variety of situations as the specific one considered here, some caution is needed for other particular cases. For instance, in the so-called steady simple shear state, there is an intrinsic coupling between inelasticity and gradients [26,27]. This implies that the shear rate and the coefficient of restitution cannot be considered as independent quantities, and the usual Chapman-Enskog procedure becomes inaccurate. This is in contrast with the situation considered here, in which the gradients are controlled by the vibrating wall and the external gravitational field. In general, each physical state should be checked to verify whether a direct expansion in the gradients is legitimate.

For the ratio between the local temperatures of the intruder and the gas, the theory predicts that it is constant in the bulk of the system. This has been confirmed by the MD simulations. The same behavior has been found in experiments [7,8] and MD simulations [6] of vibrated systems, outside the dilute and tracer limits. Nevertheless, an extension of the analysis presented in this paper [28], indicates that the ratio depends also on the local densities ratio. What happens is that this dependence is rather weak and gives corrections inside the statistical uncertainties for the values of the parameters considered in the above-mentioned works.

A qualitative discussion of the relative position of the intruder with respect to the gas was given in [12], where the crucial role played by the nonequipartition of energy was discussed. More precisely, it was shown that the intruder was above or below the center of mass of the gas depending on the sign of the pressure diffusion coefficient $D_{p}$, that, in turn, is mostly determined by the sign of $\phi-1$, as seen in Eq. (75). This is in agreement with the simulation results reported in Figs. 10-12. It can be concluded that the difference in temperature of the components of a mixture must be taken into account when studying segregation phenomena, i.e., the demix of the components when shaken. Of course, at higher densities and finite volume fraction of the components, other mechanisms $[10,29,30]$ are also important and even dominant.

It is worth mentioning that the work reported here provides another example of the accuracy of hydrodynamics to describe granular systems under rather extreme conditions of inelasticity. Although the inelasticity of the gas has been kept small in order to avoid the transversal instability, predicted by the hydrodynamic theory itself, the coefficient of inelasticity for the gas-impurity collisions has been varied over a wide range (typically between 0.3 and 0.99 ) and a reasonable good agreement between theory and simulation has been found.

\section{ACKNOWLEDGMENTS}

This research was supported by the Ministerio de Educación y Ciencia (Spain) through Grant No. FIS2005-01398 (partially financed by FEDER funds).

\section{APPENDIX: HYDRODYNAMIC PROFILES OF THE GAS}

In Ref. [21], analytical expressions for the density and temperature profiles of an open vibrated granular gas in the steady state were derived. They are given by

$$
\begin{gathered}
n(\xi)=\frac{m g_{0} \xi^{1+2 \gamma}}{C_{d} \sigma^{d-1} \sqrt{a(\alpha)}\left[A I_{\gamma}(\xi)+B K_{\gamma}(\xi)\right]^{2}}, \\
T^{1 / 2}(\xi)=\left[A I_{\gamma}(\xi)+B K_{\gamma}(\xi)\right] \xi^{\gamma},
\end{gathered}
$$

where $C_{d}=2 \sqrt{2}$ for $d=2$ and $C_{d}=\pi \sqrt{2}$ for $d=3, A$ and $B$ are constants to be determined from some boundary conditions, and $I_{\gamma}$ and $K_{\gamma}$ denote the modified Bessel functions of the first and second kind, respectively. The dimensionless length scale $\xi$ is defined by

$$
\xi=\sqrt{a(\alpha)} \int_{z}^{\infty} \frac{d z^{\prime}}{\lambda\left(z^{\prime}\right)},
$$

with $\lambda(z) \equiv\left(C_{d} n \sigma^{d-1}\right)^{-1}$ being the local mean free path. Finally, $a(\alpha)$ and $\gamma(\alpha)$ are functions of the coefficient of normal restitution, both vanishing in the elastic limit $\alpha \rightarrow 1$. Their explicit expressions are given in [21]. Note that the maximum value of $\xi$ is

$$
\xi_{0}=\frac{\sqrt{a} C_{d} \sigma^{d-1} N}{W},
$$

$N$ being the total number of particles and $W$ the length or area of the vibrating wall.

Using the hydrodynamic relation

$$
\frac{\partial p}{\partial z}=-n m g_{0},
$$

we can write $g(z)$, defined in Eq. (81), as

$$
g(z)=-\frac{1}{T}\left(m g_{0}+\frac{\zeta^{(0)}}{2 \nu} \frac{\partial T}{\partial z}\right) .
$$

The expression for $\partial T / \partial z$ is easily obtained by using Eqs. (A1)-(A3) and the properties of the modified Bessel functions [31],

$$
\frac{\partial T}{\partial z}=-\frac{2 m g_{0} \xi\left[A I_{\gamma+1}(\xi)-B K_{\gamma+1}(\xi)\right]}{A I_{\gamma}(\xi)+B K_{\gamma}(\xi)} .
$$

In this way, we have an explicit expression for the integral appearing in Eq. (82) that, therefore, can be evaluated numerically for each set of values of the parameters. Of course, this requires us to determine first the constants $A$ and $B$. In 
the results to be reported here, it has been done from the simulation data themselves, namely by fitting the position and value of the temperature minimum [21]. Another possibility that has been proven to give accurate results would be to require the hydrodynamic heat flux to vanish at infinite height [25]. Then for a granular gas with $\alpha=0.95, N=359$, $v_{W}=5$, and $W=50$, leading to $\gamma \simeq 0.021, a \simeq 0.010$, and $\xi_{0}$ $\simeq 2.065$, the values $A \simeq 5.41$ and $B \simeq 0.14$ are found. The solid lines in Fig. 1 are the fit to the hydrodynamic profiles of the gas obtained in this way.

Once $A$ and $B$ are determined, it is possible to evaluate numerically the right-hand side of Eq. (82) for each set of values of the parameters defining the mechanical properties of the intruder.
[1] J. T. Jenkins and F. Mancini, J. Appl. Mech. 54, 27 (1987).

[2] V. Garzó and J. Dufty, Phys. Rev. E 60, 5706 (1999).

[3] S. R. Dahl, C. M. Hrenya, V. Garzó, and J. W. Dufty, Phys. Rev. E 66, 041301 (2002).

[4] A. Barrat and A. Trizac, Granular Matter 4, 57 (2002).

[5] J. E. Galvin, S. R. Dhal, and C. M. Hrenya, J. Fluid Mech. 528, 207 (2005).

[6] A. Barrat and E. Trizac, Phys. Rev. E 66, 051303 (2002).

[7] K. Feitosa and N. Menon, Phys. Rev. Lett. 88, 198301 (2002).

[8] R. D. Wildman and D. J. Parker, Phys. Rev. Lett. 88, 064301 (2002).

[9] V. Garzó and J. W. Dufty, Phys. Fluids 14, 1476 (2002).

[10] J. Duran, J. Rajchenbach, and E. Clément, Phys. Rev. Lett. 70, 2431 (1993).

[11] A. P. J. Breu, H.-M. Ensner, C. A. Kruelle, and I. Rehberg, Phys. Rev. Lett. 90, 014302 (2003).

[12] J. J. Brey, M. J. Ruiz-Montero, and F. Moreno, Phys. Rev. Lett. 95, 098001 (2005).

[13] J. H. Ferziger and H. G. Karper, Mathematical Theory of Transport Processes in Gases (North-Holland, Amsterdam, 1972).

[14] A. Goldshtein and M. Shapiro, J. Fluid Mech. 282, 75 (1995).

[15] J. J. Brey, J. W. Dufty, C. S. Kim, and A. Santos, Phys. Rev. E 58, 4638 (1998).

[16] J. J. Brey and D. Cubero, in Granular Gases, edited by T. Pöschel and S. Luding, Lecture Notes in Physics (Springer-
Verlag, Berlin, 2001), p. 59.

[17] J. W. Dufty, J. J. Brey, and J. Lutsko, Phys. Rev. E 65, 051303 (2002).

[18] P. A. Martin and J. Piasecki, Europhys. Lett. 46, 613 (1999).

[19] A. Santos and J. W. Dufty, Phys. Rev. Lett. 86, 4823 (2001).

[20] S. McNamara and S. Luding, Phys. Rev. E 58, 813 (1998).

[21] J. J. Brey, M. J. Ruiz-Montero, and F. Moreno, Phys. Rev. E 63, 061305 (2001).

[22] P. Sunthar and V. Kumaran, Phys. Rev. E 64, 041303 (2001).

[23] E. Livne, B. Meerson, and P. V. Sasorov, Phys. Rev. E 65, $021302(2002)$

[24] J. J. Brey, M. J. Ruiz-Montero, F. Moreno, and R. GarcíaRojo, Phys. Rev. E 65, 061302 (2002).

[25] J. J. Brey and M. J. Ruiz-Montero, Europhys. Lett. 66, 805 (2004).

[26] N. Sela, I. Goldhirsch, and S. H. Noskowitz, Phys. Fluids 8, 2337 (1996).

[27] A. Santos, V. Garzó, and J. Dufty, Phys. Rev. E 69, 061303 (2004).

[28] J. J. Brey and M. J. Ruiz-Montero (unpublished).

[29] D. C. Hong, P. V. Quinn, and S. Luding, Phys. Rev. Lett. 86, 3423 (2001).

[30] J. T. Jenkins and D. K. Yoon, Phys. Rev. Lett. 88, 194301 (2002).

[31] Handbook of Mathematical Functions, edited by M. Abramowitz and I. A. Stegun (Dover, New York, 1965). 